\title{
Multi-objective design optimization of the combinational configuration of the upstream energy deposition and opposing jet for drag reduction in supersonic flows
}

\author{
Shengjun Ju ${ }^{a}$, Zhenxu Sun ${ }^{\mathrm{a}, *}$, Guowei Yang ${ }^{\mathrm{a}}$, Prasert Prapamonthon ${ }^{\mathrm{a}, \mathrm{b}}$, Junyuan Zhang ${ }^{\mathrm{c}}$ \\ ${ }^{a}$ Key Laboratory for Mechanics in Fluid Solid Coupling Systems Institute of Mechanics, Chinese Academy of Sciences, Beijing 100190, China \\ b Department of Aeronautical Engineering, International Academy of Aviation Industry, King Mongkut's Institute of Technology Ladkrabang, Bangkok, 10520, \\ Thailand \\ c School of Aerospace Engineering, Shenyang Aerospace University, Shenyang 110136, China
}

\section{A R T I C L E I N F O}

\section{Article history:}

Received 25 December 2019

Received in revised form 8 March 2020

Accepted 7 April 2020

Available online 12 June 2020

Communicated by Qiulin Qu

\section{Keywords:}

Optimization design

Drag reduction

Supersonic flow

Energy deposition

Opposing jet

\begin{abstract}
A B S T R A C T
Optimization design has been widely used in the supersonic vehicle design process and the drag reduction characteristic is an important objective of the optimization. The drag reduction mechanism applied to the blunt body with the combinational configuration of the upstream energy deposition and opposing jet for drag reduction has been conducted numerically. In the current study, the threedimensional coupled implicit compressible Reynolds Averaged Navier-Stokes equations and Menter's shear stress transport turbulence model are employed to simulate the flow fields around the blunt body with the combined method. The results show that in the jet-to-freestream total-pressure ratio of 0.2 and 0.4 , the drag is reduced by $47.44 \%$ and $45.96 \%$, respectively. Further, the Latin hypercube method is used for the generation of initial samples for optimization and the multi-objective design optimization algorithm coupled with the Kriging model surrogate model is applied to determine optimal flow control parameters. The drag reduction factor $R_{\mathrm{d}}$ and drag reduction effectiveness $E_{\text {eff }}$ are selected as optimization objectives. The Pareto-optimal front for the multi-objective design optimization results is acquired and there exists a challenging tradeoff between the two optimization objectives. The drag reduction factor $R_{\mathrm{d}}$ and drag reduction effectiveness $E_{\text {eff }}$ further increase as much as $28.16 \%$ and $116.47 \%$, respectively. The jet has a stronger penetration in the optimum design condition, and the findings suggest that the strategy of adding energy spot to the upstream flow field of the opposing jet can be an effective way for drag reduction.
\end{abstract}

(c) 2020 Elsevier Masson SAS. All rights reserved.

\section{Introduction}

In supersonic flight, the strong bow shock can be obviously formed in front of the blunt-body head, which can cause a significant increase in the overall drag [1]. Therefore, it's crucial to reduce the drag for the aerodynamic design of supersonic vehicles [2,3].

In order to reduce shock-drag characteristics of the vehicles, many meaningful experiments and numerical studies have been so far dedicated to flow control techniques that focus on reducing shock drag by reconstructing flow field such as aerospike [4,5], opposing jet [6,7], energy deposition [8-10] and so on. Recently, with the rapid improvement of calculating performance of the computer and the advances in numerical calculation technique, computa-

\footnotetext{
* Corresponding author.

E-mail address: sunzhenxu@imech.ac.cn (Z. Sun).
}

tional fluid dynamics (CFD) analysis has been successfully applied to simulate the supersonic flow [11-18].

The effects of spike-nosed blunt bodies on drag reduction at a Mach number of 6.8 were experimentally investigated by Crawford [19]. It was found that by adding an aerospike in the front of the blunt body, the bow shock wave can be changed into a conical shock wave. The reattachment ring and low-pressure separation zone are produced by the interaction of the conical shock wave and the reattachment shock. At zero angle of attack, the drag can be reduced by $50 \%$. However, since the cone angle of the conical shock wave produced by aerospike is small, the drag reduction effect decreases with an increasing angle of attack.

The investigation of drag reduction in supersonic flows by an opposing jet was made by Finley [20] and Hayashi [21]. Similar to the principle of aerospike drag reduction, the bow shock and the flow field structure of the blunt-body head are changed by the opposing jet. Two flow motion modes, namely, the long penetration mode (LPM) and the short penetration mode (SPM), appear with 


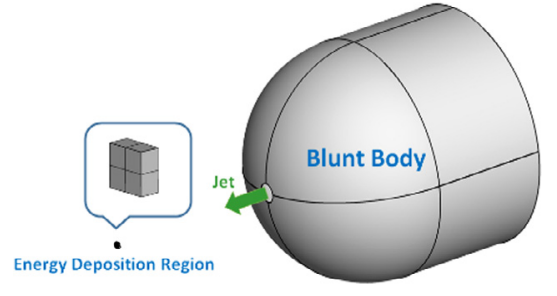

(a) Full model

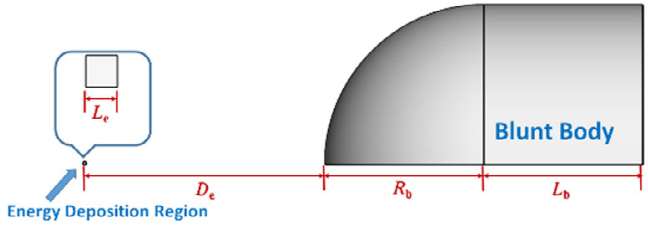

(b) Quarter model

Fig. 1. Sketch of the geometrical model.

the increase of the total-pressure ratio of the jet to freestream. But the oscillations of flow field structure caused by the opposing jet in the low total-pressure ratio affect the stability of the aircraft and the application of the opposing jet.

In recent years, energy deposition as a new active control technique for modification of the flow field has been studied and used for drag reduction by Kremeyer [22] and Azarova [8,23]. The upstream energy deposition induces a low pressure and low-density zone, which pushes the bow shock away from the blunt-body head and transforms it into oblique shock, thereby reducing the shock intensity and resulting in drag reduction in the supersonic flow. A series of the numerical simulations were performed for 15 to 45-degree cone half-angles at different Mach numbers. The result showed that the maximum drag-reduction percentage can be reached $96 \%$ in some cases, but the high cost of the energy deposition limits its application.

As a new flow control scenario, the combination of the opposing jet and the upstream energy deposition can maximize the advantages of the opposing jet and the energy deposition meanwhile avoid the disadvantages. Through the combined method, under the low-cost energy deposition, the drag can be reduced greatly and the jet flow field remains stable. The combination mode primarily has been investigated and proposed to reduce the overall drag of vehicles at supersonic/hypersonic speeds. The essence of the method is to enhance the penetrability of the opposing jet by creating a heated region, which is generated by the energy deposition, for instance, plasma, microwave, shooting combustible liquid and solid pellets.

Besides corresponding experiments, Khamooshi and Taylor et al. [24] also performed the calculation of the combination of the opposing jet and the upstream energy deposition using a twodimensional computational fluid dynamics solver with SPARK and VULCAN, for laminar and turbulent flow calculations respectively. It was found that the overall drag of representative blunt body, at the flight Mach number of 10 , can be significantly reduced by the synergistic effect of the energy deposition and the opposing jet. Furthermore, it is essential that the combination of these two methods greatly improves the stability and penetration of forward injection while the collapse of the injected plume is prevented.

Both the annular ring and swirled injections were combined with the opposing jet to conduct the drag reduction of a full threedimensional hemispherical body in the study of hypersonic flow done by Marley and Riggins [25]. Numerical simulation studies were conducted at freestream Mach numbers of 6.5 and 10 with the standard atmospheric conditions of $30 \mathrm{~km}$ altitude. The results indicated that by controlling the location of the energy deposition, the minimum drag and maximum penetration can be obtained. Besides, the stability of the jet can be maintained. The greater drag reduction can then be obtained from the combination of the upstream energy deposition and the annular ring injection.

Although the parameter effects of the combination of the opposing jet and the upstream energy deposition concept on the drag reduction have been performed by many researchers, few studies of the optimization of the flow control parameters of the combinational configuration in supersonic flows have been investigated so far. In the current study, the characteristics and alterations of flow field caused by the control of the combination of the upstream energy deposition and opposing jet configuration in supersonic flows are demonstrated and illuminated by the numerical approach and the drag reduction effect by the combined method is presented. Additionally, the relationship between the design variables and the responses is obtained by the surrogate model and the influence trend of the design variables on objective functions has been described. Furthermore, the multi-objective design optimization of the combinational configuration is considered to minimize both the drag force and drag reduction effectiveness. The main reasons for the obtained optimum condition are also analyzed. Finally, the main conclusions and recommendations for future work are drawn in the last section.

\section{Physical model and numerical algorithms}

\subsection{Physical model}

The three-dimensional blunt body model with a zero angle of attack is investigated in the current study [21]. The full and quarter geometric models are sketched in Fig. 1. The blunt body model consists of the hemispherical-nosed forebody and cylindrical main body, and there are three geometric parameters, three energy deposition parameters, and one opposing jet parameter. The geometric parameters mainly include the radius of blunt body $\left(R_{\mathrm{b}}=\right.$ $50 \mathrm{~mm})$, the length of the cylindrical main body $\left(L_{\mathrm{b}}=50 \mathrm{~mm}\right)$, and the diameter of the opposing jet orifice $\left(D_{\text {jet }}=40 \mathrm{~mm}\right)$. The previous study suggested that both the location of energy deposition and the energy deposition power have a significant impact on drag reduction [26]. For the quarter model, the distribution of the energy deposition is a uniform cube, and the three energy deposition parameters include the side length of the energy deposition cube $\left(L_{\mathrm{e}}=0.50 \mathrm{~mm}\right)$, the density of the energy deposition $\left(S_{\mathrm{e}}=200 \mathrm{~W} \cdot \mathrm{mm}^{-3}\right)$. It should be noted that ' $S_{\mathrm{e}}$ ' is the amount of energy added per unit volume $\left(Q / L_{\mathrm{e}}^{3}\right)$ in $\mathrm{W} \cdot \mathrm{mm}^{-3}$, where, $Q$ is the energy deposition power and $L_{\mathrm{e}}^{3}$ is the volume of cubic energy spot. The distance between the nose of the blunt body and the energy spot $\left(D_{\mathrm{e}}=37.50 \mathrm{~mm}\right)$. The opposing jet parameter is the total-pressure ratio of the jet to the freestream $(P R=0.4)$.

The supersonic freestream flows from left to right with a flight speed of $M a_{\infty}=3.98$. The total temperature and total pressure of the freestream are set as $T_{\infty}=397 \mathrm{~K}$ and $P_{\infty}=1.37 \mathrm{MPa}$, respectively. Air is used as the injectant against the supersonic freestream from the nose of the blunt body with the Mach number $M a_{\mathrm{j}}$ of 1.0. The total temperature of the injectant is $300 \mathrm{~K}$. Table 1 lists the corresponding flow conditions [27].

\subsection{Governing equations and turbulence model}

The three-dimensional RANS equations $[28,29]$ are solved numerically to calculate the aerodynamics of the blunt body. The governing equations are described as follows: 
Table 1

Flow conditions.

\begin{tabular}{llll}
\hline Contents & & Unit & Value \\
\hline Freestream Mach number & $M a_{\infty}$ & - & 3.98 \\
Freestream total temperature & $T_{0 \infty}$ & $\mathrm{K}$ & 397 \\
Freestream total pressure & $P_{0 \infty}$ & $\mathrm{MPa}$ & 1.37 \\
Jet spices & - & - & $\mathrm{Air}$ \\
Jet Mach number & $M a_{\mathrm{j}}$ & - & 1.0 \\
Jet total temperature & $T_{0 \mathrm{j}}$ & $\mathrm{K}$ & 300 \\
\hline
\end{tabular}

Continuity equation:

$\frac{\partial \rho}{\partial t}+\frac{\partial \rho u_{i}}{\partial x_{i}}=0$

Momentum equation:

$\frac{\partial\left(\rho u_{i}\right)}{\partial t}+\frac{\partial\left(\rho u_{i} u_{j}\right)}{\partial x_{j}}=-\frac{\partial p}{\partial x_{i}}+\frac{\partial \tau_{i j}}{\partial x_{j}}$

Energy equation:

$\frac{\partial(\rho E)}{\partial t}+\frac{\partial\left(\rho u_{j} H\right)}{\partial x_{j}}=\frac{\partial\left(u_{i} \tau_{i j}-\dot{q}_{j}\right)}{\partial x_{j}}+S_{\mathrm{e}}$

where $\rho$ is the air density, $u_{i}$ is the $i$ th component velocity, $H$ is the total enthalpy, $p$ is the pressure, and $E$ is the total energy. The shear stress term $\tau_{\mathrm{ij}}$ is the sum of a laminar and a turbulent component. The laminar viscosity is calculated by the Sutherland formula, and the turbulent viscosity is calculated by the SST $k-\omega$ turbulence model. The previous study has shown that the heating effect can be reasonably approximated via an energy addition rate with uniform distribution [30]. The present simulations employ the uniform distribution defined in Eq. (4). Here, $Q$, which describes the energy deposition power, is defined as the product of cubic energy spot volume $V_{\mathrm{e}}$ and the energy deposition density $S_{\mathrm{e}}$. It is noted that $S_{\mathrm{e}}$ stands for the source term of the energy deposition (also known as the density of the energy deposition), which equals 0 when the case without energy deposition is computed $[31,32]$.

$S_{\mathrm{e}}=\frac{Q}{V_{\mathrm{e}}}$

The pressure $p$ is acquired from the state equation for perfect gas:

$p=\rho R T=(\gamma-1)\left[\rho E-\frac{1}{2} \rho\left(u^{2}+v^{2}+w^{2}\right)\right]$

$E=e+\frac{1}{2}\left(u^{2}+v^{2}+w^{2}\right) \quad H=e+\frac{p}{\rho}$

where $u, v, w$ is the velocity in Cartesian coordinates. $T$ is the temperature and $e$ is the internal energy, and $\gamma$ denotes the specific heat ratio.

The SST $k-\omega$ turbulence model was developed by Menter [33] with a significant influence from the original $k-\omega$ model proposed by Wilcox and the standard $k-\varepsilon$ model. This model uses the $k-\omega$ model near the wall surface and the $k-\varepsilon$ model in the free shear flow region. Therefore, it is widely used to simulate hypersonic flows in engineering applications. The model is composed of twotransport equations of the turbulent kinetic energy $k$ and the specific dissipation rate $\omega$.

Transport equation of turbulent kinetic energy $k$ :

$$
\begin{aligned}
\frac{\partial(\rho k)}{\partial t}+\frac{\partial\left(\rho u_{j} k\right)}{\partial x_{j}}= & \frac{\partial}{\partial x_{j}}\left[\left(\mu_{L}+\sigma_{k} \mu_{T}\right) \frac{\partial k}{\partial x_{j}}\right] \\
& +P_{k}-\beta^{*} \rho \omega k
\end{aligned}
$$

Transport equation of the specific dissipation rate of turbulence $\omega$ :

$$
\begin{aligned}
\frac{\partial(\rho \omega)}{\partial t}+\frac{\partial\left(\rho u_{j} \omega\right)}{\partial x_{j}}= & \frac{\partial}{\partial x_{j}}\left[\left(\mu_{L}+\sigma_{\omega} \mu_{T}\right) \frac{\partial \omega}{\partial x_{j}}\right]+P_{\omega}-\beta \rho \omega^{2} \\
& +2\left(1-f_{1}\right) \frac{\rho \sigma_{\omega 2}}{\omega} \frac{\partial k}{\partial x_{j}} \frac{\partial \omega}{\partial x_{j}}
\end{aligned}
$$

where $P_{k}$ and $P_{\omega}$ denote production terms of the turbulent kinetic energy $k$ and the specific dissipation rate of turbulence $\omega$, respectively. $\mu_{L}$ and $\mu_{T}$ are the laminar and turbulent viscosity, respectively. $f_{1}$ is auxiliary functions, whereas $\sigma_{\mathrm{k}}, \sigma_{\omega}, \beta$ and $\beta^{*}$ are model constants $[33,34]$.

\subsection{Discretization and boundary conditions}

The governing equations discussed above are discretized by the finite volume method (FVM) in the multi-block structured grid system. Inviscid fluxes are discretized by Roe flux-difference scheme with second-order Monotone Upstream-centered Schemes for Conservation Laws (MUSCL) reconstruction and minmod limiter [31]. The viscous fluxes are discretized by the second-order central difference scheme. The implicit Lower-Upper Symmetric Gauss-Seidel (LUSGS) scheme is employed for time advancement. The CourantFriedrichs-Levy (CFL) number is kept at 0.75 with proper underrelaxation factors to ensure stability [32].

The condition of the non-slip wall with the zero gradient and isothermal temperature of $295 \mathrm{~K}$ is employed for the wall surface [34]. The velocity normal to the symmetry plane is zero. In addition, the freestream levels of $k, \omega$ and $\mu_{\mathrm{T}}$ at far-field boundaries for the SST $k-\omega$ turbulence model are specified as reported [33]:

$$
\begin{aligned}
& \frac{k_{\infty}}{a_{\infty}^{2}}=9 \times 10^{-9}, \quad \frac{\omega_{\infty}}{\left(\rho_{\infty} a_{\infty}^{2}\right) / \mu_{\mathrm{L} \infty}}=1 \times 10^{-6}, \\
& \frac{\mu_{\mathrm{T}, \infty}}{\mu_{\mathrm{L}, \infty}}=0.009
\end{aligned}
$$

On the solid wall, the values of $k$ and $\omega$ are determined, respectively, as:

$k_{\text {wall }}=0, \quad \omega_{\text {wall }}=\frac{60 \mu_{1}}{0.075 \rho_{1}\left(d_{1}\right)^{2}}$

where $\mu_{1}$ and $\rho_{1}$ are the laminar viscosity and density, respectively. $d_{1}$ is the distance between the cell centers of first grids near the solid wall to the solid wall [34].

\subsection{Grid sensitivity analysis and numerical validation}

The numerical results obtained by the CFD code are validated with experimental data performed by Hayashi $[21,27]$ in terms of the pressure distribution. The jet total pressure ratio $P R$ is set as 0.4 and the other freestream and jet flow conditions are given in Table 1.

To prove the grid independence of numerical calculation, three configurations with different computational grids are employed to perform the grid sensitivity study in the flow field of the blunt body with the opposing jet, namely, coarse grid, medium grid, and fine grid. Two structured grid blocks are generated in this study. The grid resolution of the energy deposition zone is $10 \times 19 \times 10$ with the $0.05 \mathrm{~mm}$ size. The structured meshes of the medium grid are shown in Fig. 2. Besides, for the different energy deposition locations, the size of the domain and the grid resolution remain constant in the energy deposition zone, as shown in Fig. 3.

The grid Reynolds number $R e_{\text {grid }}$, which is determined by the first grid spacing of the solid wall in the normal direction, can be calculated as Eq. (11). 
Table 2

Grid system information.

\begin{tabular}{llll}
\hline & Coarse grid & Medium grid & Fine grid \\
\hline First cell height, mm & 0.0017 & 0.0011 & 0.0006 \\
$R e_{\text {grid }}$ & 67.8 & 45.2 & 22.6 \\
First block grid resolution & $319 \times 85 \times 131$ & $332 \times 92 \times 161$ & $341 \times 94 \times 191$ \\
Second block grid resolution & $319 \times 71 \times 31$ & $332 \times 81 \times 41$ & $341 \times 91 \times 51$ \\
Num. of grid & $4.25 \times 10^{6}$ & $6.02 \times 10^{6}$ & $7.70 \times 10^{6}$ \\
\hline
\end{tabular}

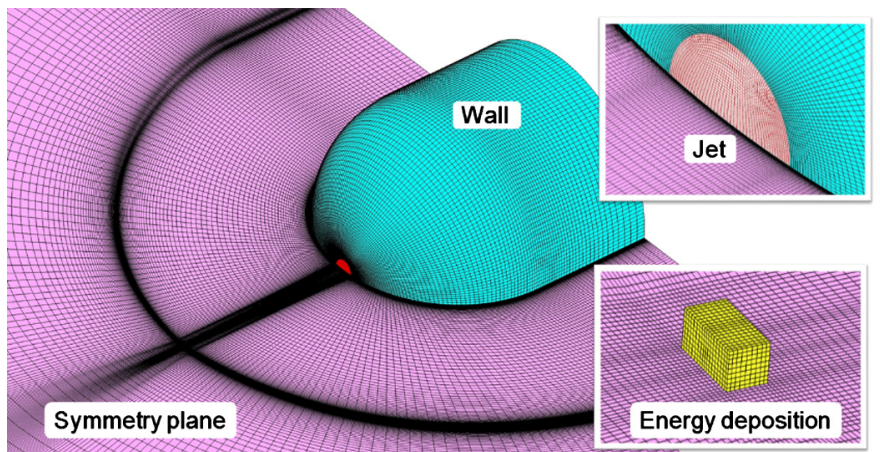

Fig. 2. The structured meshes of medium grid.
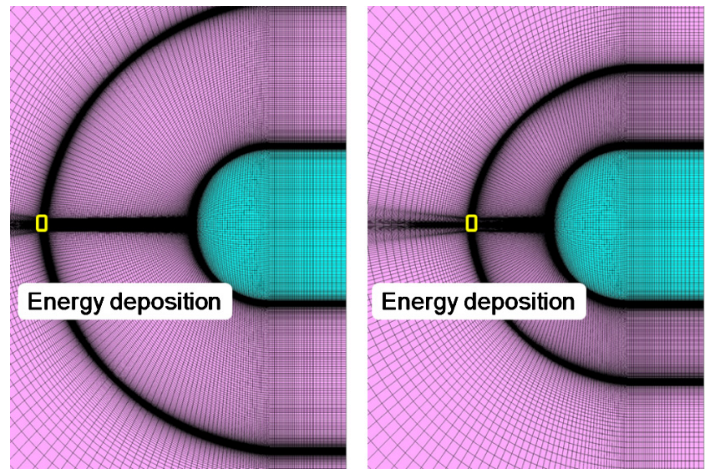

Fig. 3. The structured meshes of different energy deposition location.

$R e_{\text {gird }}=\frac{\rho_{\infty} u_{\infty} d}{\mu_{\infty}}$

where $\rho_{\infty}, u_{\infty}$ and $\mu_{\infty}$ are the density, velocity, and viscosity of the freestream, respectively, and $d$ is the first cell height in the direction normal to the surface. The cell height of the first row in the coarse grid, medium grid, and fine grid are $0.0017 \mathrm{~mm}$, $0.0011 \mathrm{~mm}$, and $0.0006 \mathrm{~mm}$, respectively. Details of grid system information are shown in Table 2.

Additionally, $\mathrm{Y}^{+}$is another main factor of the grid analysis, which is important in turbulence modeling to determine the first cell height in the direction normal to the wall surface. In this study, the first cell height is refined to obtain the $\mathrm{Y}^{+}$value of 1.0 approximately for confirming the numerical precision.

The comparison of the computed pressure distributions of the three-configurations and experimental results are presented in Fig. 4. It is found that the numerical pressure distributions agree well with the experimental results. At the same time, the results obtained by the coarse grid and medium grid do not exhibit any significant differences to those obtained by the fine grid so that the numerical precision and the computational cost are guaranteed. Therefore, the medium grid is used for the following study.

The Mach number contours calculated by the numerical approach are compared with the Schlieren images obtained from experiments in Fig. 5. As shown, the distinct structure details, such as the bow shock, barrel shock, reattachment shock, and the Mach

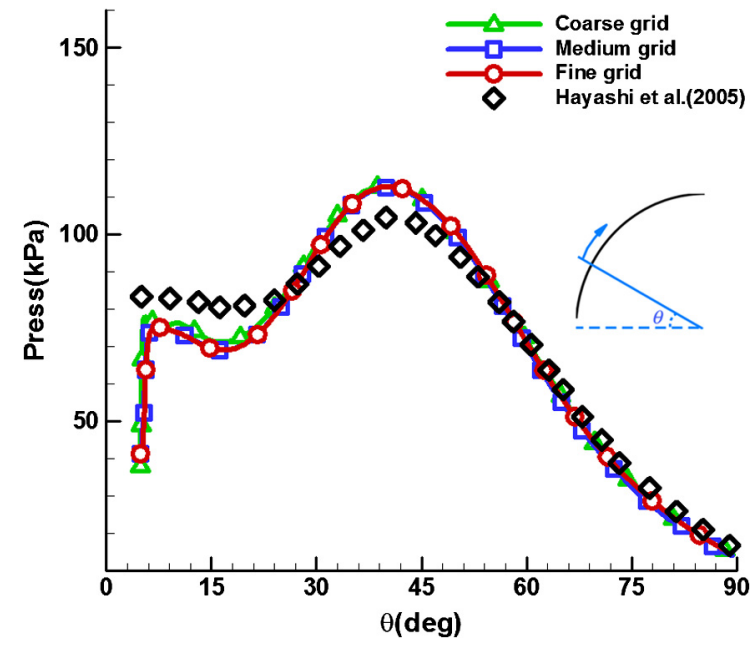

Fig. 4. Comparing results between numerical simulation and experiment.

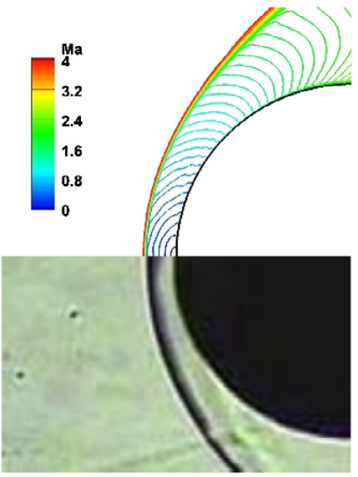

(a) No jet

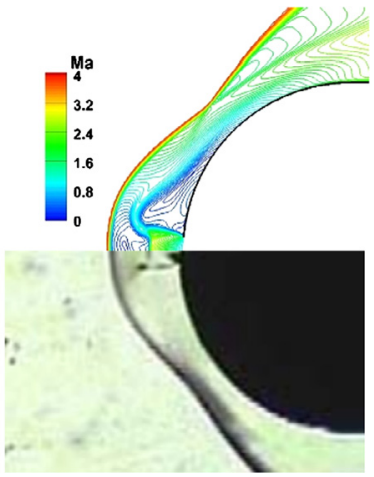

(b) $P R=0.4$
Fig. 5. Experimental Schlieren image (upper) versus calculated Mach number contours (lower). (For interpretation of the colors in the figure(s), the reader is referred to the web version of this article.)

disk, can be captured by the predicted approach. The calculated jet flow field structure is in accord with the experimental Schlieren photograph well. Therefore, the present numerical approach can be used to predict complex flow structures acceptably and reliably for the following investigations.

\section{Comparison of the drag reduction efficiency}

In order to obtain the drag reduction efficiency of different flow control methods, the calculated features including flow structures, pressure distributions, non-dimensional shock standoff distances $(\Delta)$, and drag reduction factors $\left(R_{\mathrm{d}}\right)$ should be compared at the jet total pressure ratios $P R$ of 0.2 , and 0.4 respectively. The freestream and jet conditions of the numerical calculation are presented in Table 1 . The non-dimensional shock standoff distances $(\Delta)$ is defined as the centerline distance from the blunt-body shock front to the leading edge of the blunt body divided by the blunt-body diameter [24]. The drag reduction factors $R_{\mathrm{d}}$ is defined as Eq. (12). 


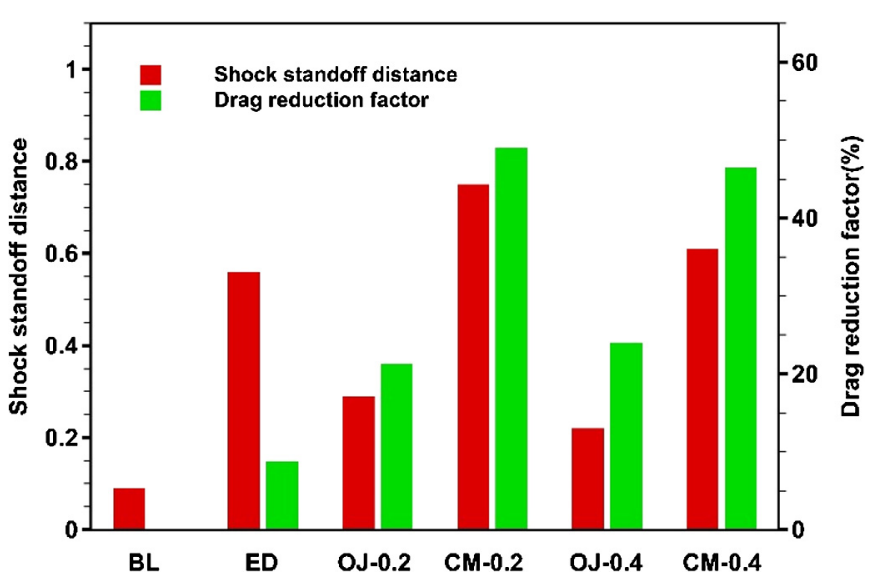

Fig. 6. Drag reduction efficiency by different control modes.

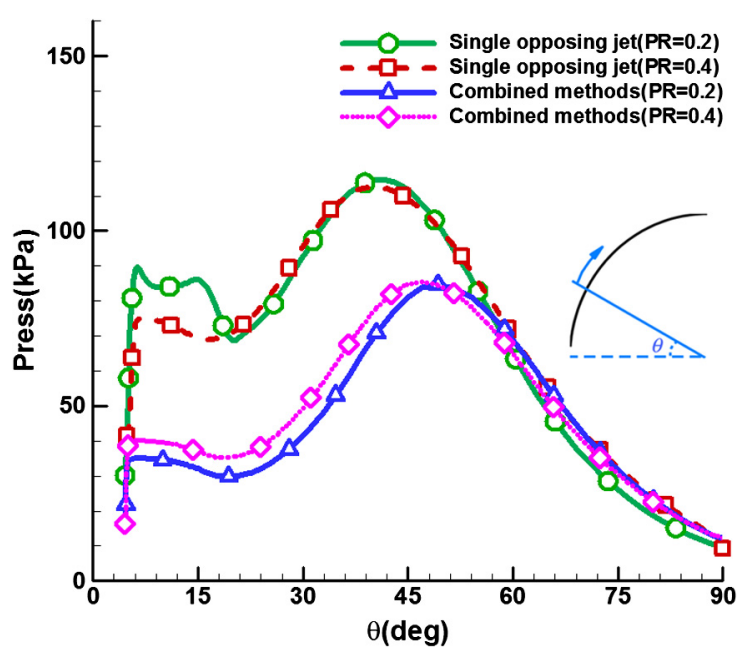

Fig. 7. Centerline surface pressure distributions.

$R_{\mathrm{d}}=\frac{C D_{\mathrm{ref}}-C D_{\mathrm{mod}}}{C D_{\mathrm{ref}}}$

where $C D_{\text {ref }}$ and $C D_{\text {mod }}$ are the drag coefficient of baseline and modification, respectively.

Drag reduction efficiency by different control modes is summarized in Fig. 6. The $x$-axis includes the baseline (no flow-field modification or injection) (BL), single energy deposition (ED), single opposing jet at $P R=0.2(\mathrm{OJ}-0.2)$, the combined method at $P R=0.2(\mathrm{CM}-0.2)$, single opposing jet at $P R=0.4(\mathrm{OJ}-0.4)$, the combined method at $P R=0.4(\mathrm{CM}-0.4)$. For both pressure ratios of $P R=0.2$ and $P R=0.4$, a general trend of the drag reduction can be noticed and the use of the combined method provides a greater drag reduction. It can be seen that the maximum of $49.04 \%$ drag reduction and 0.75 shock standoff distance can be achieved in the case of $P R=0.2$ with the method of combining the upstream energy deposition and opposing jet configuration.

Moreover, the comparisons of surface pressure variations with the opposing jet only and the combined method at $P R=0.2$ and $P R=0.4$ are shown in Fig. 7. It can be seen that for the opposing jet only, the case of $P R=0.4$ gives the lower values of surface pressures than those of $P R=0.2$. However, for the combined method, the lowest value of surface pressures appears in the case of $P R=0.2$ rather than $P R=0.4$. The superiority of the opposing jet coupled with the energy deposition upstream of the blunt body is clearly observed.

The flow structures of the blunt model with the combined method at $P R=0.2$ and $P R=0.4$ are illustrated in Fig. 8. As a mutual influence of the supersonic mainstream, the opposing jet,
Table 3

Range of variables.

\begin{tabular}{lllll}
\hline Variable & $D_{\mathrm{e}} / \mathrm{mm}$ & $L_{\mathrm{e}} / \mathrm{mm}$ & $S_{\mathrm{e}} / \mathrm{W} \cdot \mathrm{mm}^{-3}$ & $P R$ \\
\hline Lower limit & 26.25 & 0.350 & 200 & 0.200 \\
Upper limit & 48.75 & 0.650 & 600 & 0.600
\end{tabular}

and the upstream energy deposition, the bow shock disappearing completely, the oblique shock is formed by compression waves and the barrel shock is generated in the opposing jet column. At the pressure ratio $P R=0.2$, due to the upstream energy deposition, the opposing jet directly penetrates the supersonic mainstream and reaches the position of energy deposition. However, at the pressure ratio $P R=0.4$, Mach disk generated by the interaction of the opposing jet and the mainstream is stagnated between the position of the energy deposition and the head of the blunt body, and two distinct recirculation regions are recognized.

\section{Optimization design process}

The energy consumption ratio and cost of the energy deposition are very important to measure drag reduction performance. The maximum drag reduction factors $R_{\mathrm{d}}$ and drag reduction effectiveness $E_{\text {eff }}$ should be considered when selecting optimization objectives [9,35]. The non-dimensional objective function of $E_{\text {eff }}$ is defined as the ratio of the amount of profit gained by drag reduction to the amount spent for the energy deposition.

$E_{\text {eff }}=\frac{\left(C D_{\text {ref }}-C D_{\text {mod }}\right) V_{\infty}}{Q}$

where, $V_{\infty}$ and $Q$ are the velocity and the power of energy deposition, respectively.

There are four flow control parameters included in selecting design variables. The distance between the nose of blunt body and energy spot, $D_{\mathrm{e}}$, the side length of the energy deposition cube, $L_{\mathrm{e}}$, the density of the energy deposition, $S_{\mathrm{e}}$, and the jet total-pressure ratio, $P R$ are selected as the design variables. The design space of the variables is listed in Table 3.

The optimum flow control parameters of the combined method mentioned above are obtained by the multi-objective design optimization algorithm coupled with the Kriging surrogate model [36,37]. The nondominated sorting genetic algorithm (NSGA II) [38,39], a famous multi-objective design optimization algorithm, is of good performance of global optimization performance and widely used in design optimization of nozzle [31], the cavity flame holder [40], the hypersonic spiked bodies [41,42] and the combinational novel cavity [36] and so on. The sub-population size, the number of generations, and the crossover probability are set as 40 , 100 , and 0.9 respectively. The flowchart of the optimization design process is shown in Fig. 9.

The Latin hypercube method [43] benefits from its better performance of homogeneity and space-filling efficiency $[44,45]$, which is adopted to generate initial samples in the current study and 40 samples of four design parameters are generated in the design space. Both design variable arrangements and objective function results by numerical calculation are shown in Table 4 .

The fidelity of the optimization design depends highly on the accuracy of the surrogate model. A set of 5 test samples are randomly selected in the design variables ranges for surrogate model validation (see Table 5). The coefficient of determination $\left(\mathrm{R}^{2}\right)[46,47]$ and root mean square error (RMSE) [48] are provided to measure the accuracy and reliability of the surrogate model approach, as seen in Eq. (14).

$\mathrm{R}^{2}=1-\frac{\sum_{i=1}^{n}\left(y_{i}-\hat{y}_{i}\right)^{2}}{\sum_{i=1}^{n}\left(y_{i}-\bar{y}_{i}\right)^{2}} \quad \mathrm{RMSE}=\sqrt{\frac{\sum_{i=1}^{n}\left(y_{i}-\hat{y}_{i}\right)^{2}}{n}}$ 


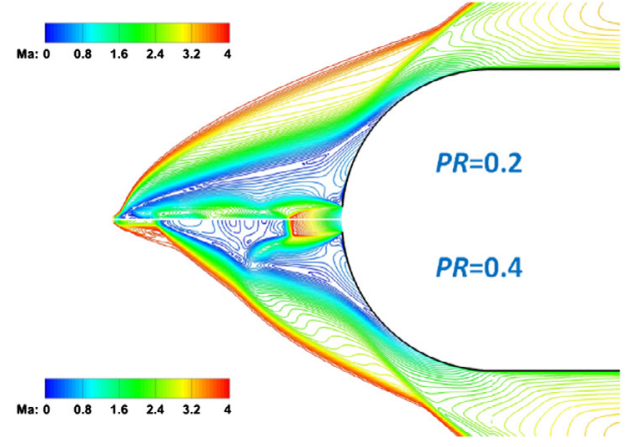

(a)Mach number contours

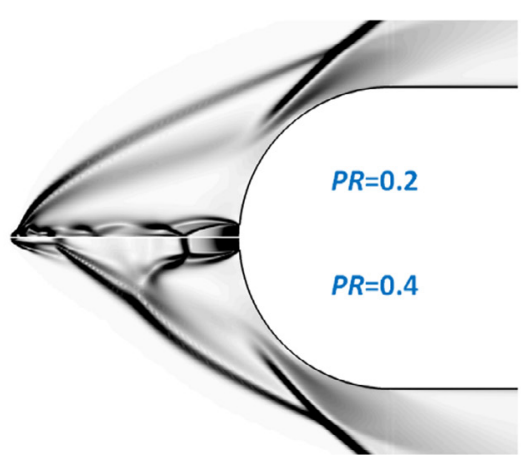

(b)Density gradient magnitudes

Fig. 8. Comparisons of flow field structure for different cases.

Table 4

Sampling points and CFD results.

\begin{tabular}{|c|c|c|c|c|c|c|}
\hline \multirow{2}{*}{$\begin{array}{l}\text { Sample } \\
\text { number }\end{array}$} & \multicolumn{4}{|c|}{ Design variable } & \multicolumn{2}{|c|}{ Objective function } \\
\hline & $D_{\mathrm{e}} / \mathrm{mm}$ & $L_{\mathrm{e}} / \mathrm{mm}$ & $S_{\mathrm{e}} / \mathrm{W} \cdot \mathrm{mm}^{-3}$ & $P R$ & $R_{\mathrm{d}} / \%$ & $E_{\text {eff }}$ \\
\hline 1 & 26.25 & 0.419 & 354 & 0.497 & 43.58 & 581.41 \\
\hline 2 & 26.83 & 0.512 & 497 & 0.426 & 41.77 & 218.47 \\
\hline 3 & 27.40 & 0.458 & 344 & 0.303 & 35.69 & 376.49 \\
\hline 4 & 27.98 & 0.550 & 292 & 0.559 & 48.07 & 344.28 \\
\hline 5 & 28.55 & 0.581 & 241 & 0.364 & 40.09 & 295.41 \\
\hline 6 & 29.13 & 0.627 & 415 & 0.282 & 38.73 & 131.78 \\
\hline 7 & 29.70 & 0.504 & 528 & 0.231 & 43.10 & 222.11 \\
\hline 8 & 30.30 & 0.389 & 508 & 0.344 & 41.51 & 484.88 \\
\hline 9 & 30.88 & 0.642 & 426 & 0.487 & 49.39 & 152.22 \\
\hline 10 & 31.45 & 0.519 & 487 & 0.600 & 53.11 & 271.10 \\
\hline 11 & 32.03 & 0.381 & 508 & 0.538 & 50.91 & 631.49 \\
\hline 12 & 32.60 & 0.465 & 200 & 0.446 & 45.02 & 776.86 \\
\hline 13 & 33.18 & 0.350 & 323 & 0.385 & 43.06 & 1081.74 \\
\hline 14 & 33.75 & 0.542 & 282 & 0.200 & 44.38 & 343.32 \\
\hline 15 & 34.33 & 0.596 & 579 & 0.354 & 47.40 & 134.39 \\
\hline 16 & 34.90 & 0.404 & 210 & 0.241 & 48.05 & 1208.98 \\
\hline 17 & 35.48 & 0.527 & 374 & 0.395 & 46.30 & 294.47 \\
\hline 18 & 36.05 & 0.435 & 333 & 0.579 & 53.72 & 683.72 \\
\hline 19 & 36.63 & 0.427 & 405 & 0.210 & 47.47 & 524.18 \\
\hline 20 & 37.20 & 0.612 & 251 & 0.508 & 51.40 & 311.61 \\
\hline 21 & 37.80 & 0.481 & 549 & 0.456 & 51.09 & 291.28 \\
\hline 22 & 38.38 & 0.619 & 559 & 0.549 & 54.81 & 143.70 \\
\hline 23 & 38.95 & 0.442 & 600 & 0.272 & 54.18 & 363.06 \\
\hline 24 & 39.53 & 0.581 & 446 & 0.221 & 51.16 & 203.69 \\
\hline 25 & 40.10 & 0.635 & 262 & 0.313 & 56.31 & 292.58 \\
\hline 26 & 40.68 & 0.365 & 456 & 0.374 & 46.99 & 734.83 \\
\hline 27 & 41.25 & 0.558 & 395 & 0.590 & 56.99 & 289.40 \\
\hline 28 & 41.83 & 0.650 & 436 & 0.405 & 49.85 & 144.85 \\
\hline 29 & 42.40 & 0.496 & 231 & 0.323 & 58.35 & 719.34 \\
\hline 30 & 42.98 & 0.358 & 272 & 0.467 & 41.14 & 1149.68 \\
\hline 31 & 43.55 & 0.396 & 477 & 0.569 & 56.30 & 660.23 \\
\hline 32 & 43.55 & 0.489 & 221 & 0.528 & 52.31 & 706.38 \\
\hline 33 & 44.70 & 0.373 & 303 & 0.262 & 56.79 & 1255.54 \\
\hline 34 & 45.30 & 0.565 & 569 & 0.333 & 61.67 & 208.63 \\
\hline 35 & 45.88 & 0.473 & 385 & 0.436 & 49.73 & 424.43 \\
\hline 36 & 46.45 & 0.450 & 467 & 0.251 & 57.38 & 469.09 \\
\hline 37 & 47.03 & 0.412 & 590 & 0.415 & 64.53 & 546.09 \\
\hline 38 & 47.60 & 0.535 & 538 & 0.508 & 55.60 & 235.34 \\
\hline 39 & 48.18 & 0.604 & 313 & 0.477 & 66.79 & 337.25 \\
\hline 40 & 48.75 & 0.573 & 364 & 0.292 & 61.24 & 310.95 \\
\hline
\end{tabular}

where, $y_{i}, \hat{y}_{i}$, and $\bar{y}_{i}$ are the test sampling value, the corresponding response value of the Kriging surrogate model, and the average of the test values, respectively.

The values of $\mathrm{R}^{2}$ are 0.928 and 0.908 for the two optimization objectives ( $E_{\text {eff }}$ and $R_{\mathrm{d}}$ are shown in Table 6), respectively. Both of those exceed the acceptance of the threshold value of 0.90 . In addition, the values of RMSE are 0.095 and 0.126 for the $R_{\mathrm{d}}$ and $E_{\text {eff }}$, respectively. Both of those are lower than the acceptance of the threshold value of 0.2 . Therefore, it is suggested that the modeling accuracy of the Kriging surrogate is adequate to integrate with optimization design.
The correlations are obtained by the surrogate model, thereby indicating the relationship between the design variables and the responses, as shown in Figs. 10 and 11. The results show that the drag reduction factor $R_{\mathrm{d}}$ increases with the increase of the design variable $D_{\mathrm{e}}, L_{\mathrm{e}}$ and $S_{\mathrm{e}}$. However, the drag reduction factor $R_{\mathrm{d}}$ decreases first and then increases with the increase of the design variable $P R$, and there exists a penetration mode of the transformation and this implication is also realized by Fig. 8. It is observed that the drag reduction effectiveness $E_{\text {eff }}$ increases significantly with the decrease of the design variable $L_{\mathrm{e}}$ and $S_{\mathrm{e}}$. Therefore, the design variables of $L_{\mathrm{e}}$ and $S_{\mathrm{e}}$ have a significant impact on drag re- 
Table 5

Test sample points and CFD results.

\begin{tabular}{llllllll}
\hline \multirow{2}{*}{$\begin{array}{l}\text { Test } \\
\text { sample }\end{array}$} & \multicolumn{2}{l}{ Design variable } & & \multicolumn{2}{c}{ Objective function } \\
\cline { 2 - 4 } & $D_{\mathrm{e}} / \mathrm{mm}$ & $L_{\mathrm{e}} / \mathrm{mm}$ & $S_{\mathrm{e}} / \mathrm{W} \cdot \mathrm{mm}^{-3}$ & $P R$ & & $R_{\mathrm{d}} / \%$ & $E_{\mathrm{eff}}$ \\
\hline 1 & 27.85 & 0.472 & 452 & 0.298 & & 36.66 & 268.64 \\
2 & 28.23 & 0.419 & 433 & 0.522 & & 46.74 & 509.61 \\
3 & 35.55 & 0.439 & 252 & 0.519 & & 50.73 & 826.72 \\
4 & 42.80 & 0.514 & 470 & 0.568 & & 57.73 & 314.54 \\
5 & 46.90 & 0.439 & 317 & 0.314 & & 61.11 & 790.68 \\
\hline
\end{tabular}

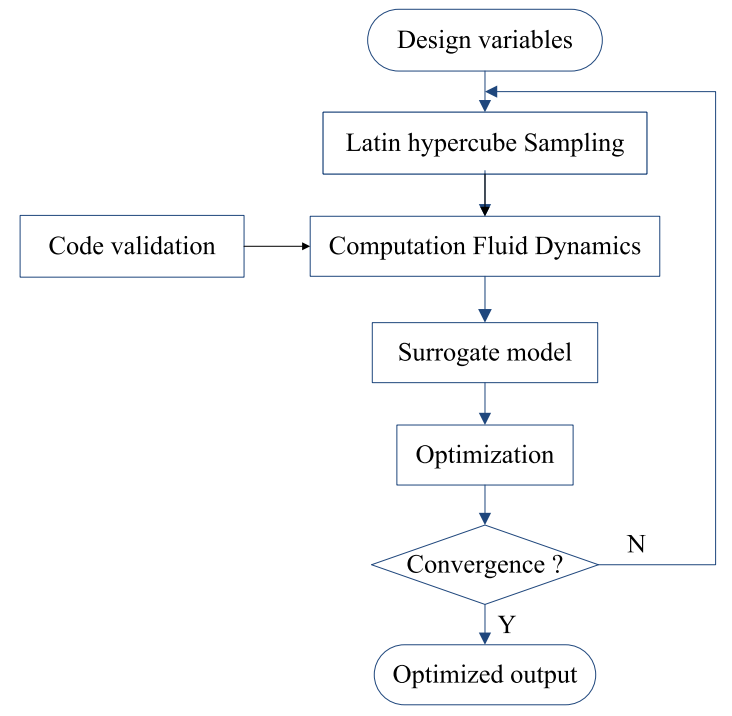

Fig. 9. Flowchart of optimization design process.

Table 6

Results of modeling precision.

\begin{tabular}{lll}
\hline Objective function & $\mathrm{R}^{2}$ & RMSE \\
\hline drag reduction factors $R_{\mathrm{d}}$ & 0.928 & 0.095 \\
drag reduction effectiveness $E_{\text {eff }}$ & 0.908 & 0.126 \\
\hline
\end{tabular}

duction effectiveness, and the size and the density of the energy deposition are more important for drag reduction efficiency. The influences of the other two design variables, namely $D_{\mathrm{e}}$ and $P R$, are not substantial for drag reduction effectiveness $E_{\text {eff }}$.

\section{Optimization design results}

The feasible results of drag reduction factors $R_{\mathrm{d}}$ and drag reduction effectiveness $E_{\text {eff }}$ during the optimization history are shown by scatter plots in Fig. 12. The Pareto-optimal front [49] obtained through the optimization design method can be clearly recognized [50]. It is observed that the drag reduction factor $R_{\mathrm{d}}$ increases with the decrease in drag reduction effectiveness $E_{\text {eff. This }}$ implies that two optimization objectives in this design process are inconsistent. Hence, a compromise between the two objectives is needed.

The weighting method, as the most typical normalized method, is applied to choose the optimized case from the Pareto-optimal front for detailed performance analysis. The expression is as follows:

$f=a_{1}\left(\frac{R_{\mathrm{d}}-R_{\mathrm{dmin}}}{R_{\mathrm{dmax}}-R_{\mathrm{dmin}}}\right)^{2}+a_{2}\left(\frac{E_{\mathrm{eff}}-E_{\text {eff min }}}{E_{\text {eff max }}-E_{\text {eff min }}}\right)^{2}$

where $f$ is the objective function, $a_{1}$ and $a_{2}$ are weighting coefficients and set to 1.0 , and $R_{\mathrm{dmin}}, R_{\mathrm{dmax}}, E_{\text {eff min }}$ and $E_{\text {eff } \max }$ are the minimum value and maximum value of drag reduction factor
$R_{\mathrm{d}}$ and drag reduction effectiveness $E_{\text {eff }}$ in feasible results, respectively.

The optimal parametric combinations are selected and given in Table 7. The optimized performances obtained by the optimization design approach are in good agreement with the computational fluid dynamics (CFD) results. This indicates the accuracy and reliability of the present optimization approach and reflects the similarity of predicted and actual optimized results. For the optimized configuration, the drag reduction factor $R_{\mathrm{d}}$ further increases by $28.16 \%$, whereas the drag reduction effectiveness $E_{\text {eff }}$ also further increases by $116.47 \%$. It should be noted that the optimized performances coincide well with the numerical predictions, which show the validity and accuracy of the optimal design method. At the same time, for the optimized design, the location for the region of the energy deposition appears to be the maximized distance between the nose of blunt body and the energy spot.

The centerline distributions of the surface pressure under the condition between the original and optimized control configurations are compared in Fig. 13. It is noticeable that the pressure distribution on the surface shows a significant difference, especially in the region from $\theta=40^{\circ}$ to $\theta=60^{\circ}$ which the surface pressure of the optimized design is much lower than that of the original design.

A comparison of the flow field structure between the original and optimized control configurations is shown in Fig. 14. It is found that the shock standoff distance of the optimized design is much longer than that of the original configuration, as well as the size of the separation zone. From the comparison of Mach number contours, it should be noted that the jet expands intensively and the jet has a stronger penetration in the optimized control configuration when compared to the jet in the original design.

In optimum condition, with increasing the design variable $D_{\mathrm{e}}$, the size of energy spot $L_{\mathrm{e}}$ and the density of the energy deposition $S_{\mathrm{e}}$ can increase the penetration distance of the opposing jet and reduce the reattachment shock intensity simultaneously. Therefore, the drag reduction further decreases. However, the nondimensional objective function of $E_{\text {eff }}$ is inversely proportional to the design variables of $L_{\mathrm{e}}$ and $S_{\mathrm{e}}$. Hence, in the optimized control configuration, both the design variable of $L_{\mathrm{e}}$ and $S_{\mathrm{e}}$ are greater but not reach the maximum. It is noteworthy that, with increasing the design variable of $P R$, the long penetration mode of the jet is transformed into the short penetration mode, and the drag decreases first and then increases. As a result, the optimum design variable of $P R$ is in the middle of the lower and upper limits.

\section{Conclusions}

In this paper, the comparisons of the flow field and drag characteristics of the blunt-body model with three configurations (namely the energy deposition, the opposing jet, and the combination of the upstream energy deposition and opposing jet configuration) are presented. The parameter effects of the combined control mode are demonstrated and analyzed by variance analysis method coupled with orthogonal experimental design. Interesting findings are summarized as follows: 


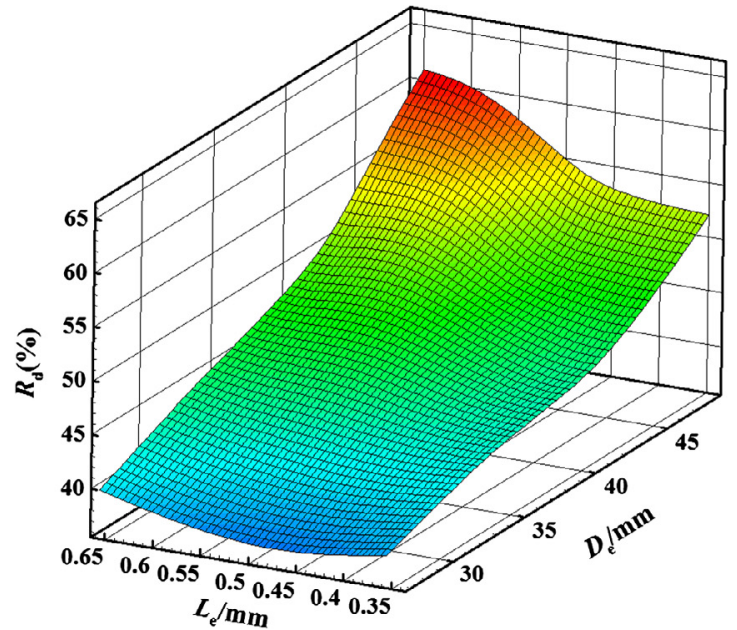

(a) $R_{\mathrm{d}}$ versus $D_{\mathrm{e}}$ and $L_{\mathrm{e}}$

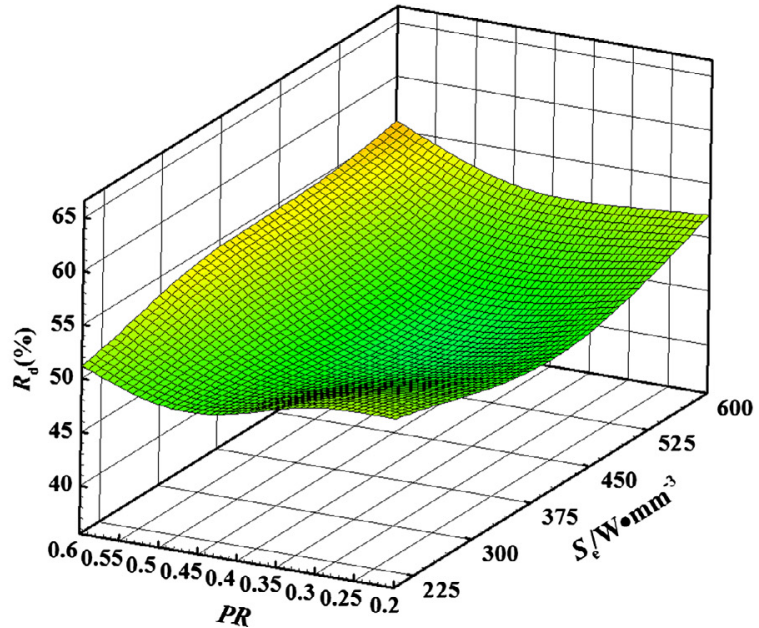

(b) $R_{\mathrm{d}}$ versus $S_{\mathrm{e}}$ and $P R$

Fig. 10. Surrogate model of the drag reduction factor $R_{\mathrm{d}}$.

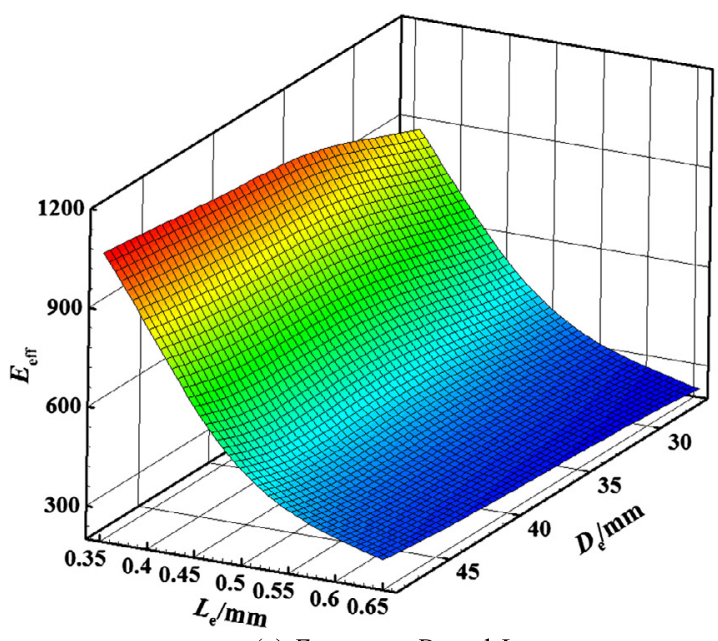

(a) $E_{\text {eff }}$ versus $D_{\mathrm{e}}$ and $L_{\mathrm{e}}$

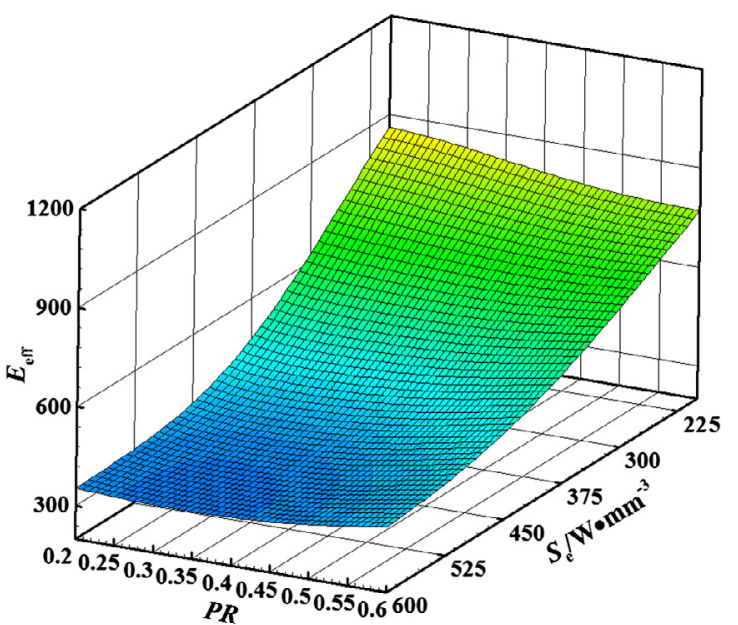

(b) $E_{\text {eff versus }} S_{\mathrm{e}}$ and $P R$

Fig. 11. Surrogate model of the drag reduction effectiveness $E_{\text {eff }}$.

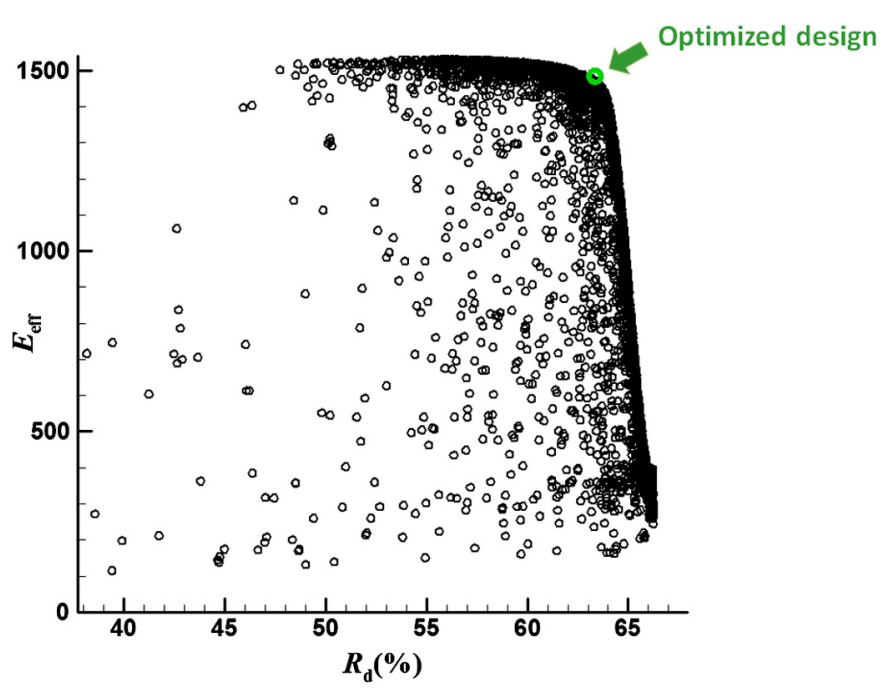

Fig. 12. Feasible results during the optimization history.

(1) An active drag reduction technique based on the combinational strategy of the steady energy deposition and opposing jet in the supersonic stream is presented. The proposed nu- merical method can be used to predict the three-dimensional flow field of the combinational energy deposition and opposing jet configuration. 
Table 7

Comparisons of the optimized performances with the original performances.

\begin{tabular}{|c|c|c|c|c|c|c|c|c|}
\hline & \multicolumn{4}{|c|}{ Design variable } & \multicolumn{2}{|c|}{ Objective function } & \multicolumn{2}{|l|}{$\Delta(\%)$} \\
\hline & $D_{\mathrm{e}} / \mathrm{mm}$ & $L_{\mathrm{e}} / \mathrm{mm}$ & $S_{\mathrm{e}} / \mathrm{W} \cdot \mathrm{mm}^{-3}$ & $P R$ & $R_{\mathrm{d}} / \%$ & $E_{\text {eff }}$ & $R_{\mathrm{d}}$ & $E_{\text {eff }}$ \\
\hline Original & 37.50 & 0.5 & 200 & 0.2 & 49.04 & 682.45 & - & - \\
\hline Optimized & 48.75 & 0.384 & 244 & 0.311 & 63.19 & 1582.65 & $28.85 \%$ & $131.91 \%$ \\
\hline CFD & 48.75 & 0.384 & 244 & 0.311 & 62.85 & 1477.32 & $28.16 \%$ & $116.47 \%$ \\
\hline
\end{tabular}

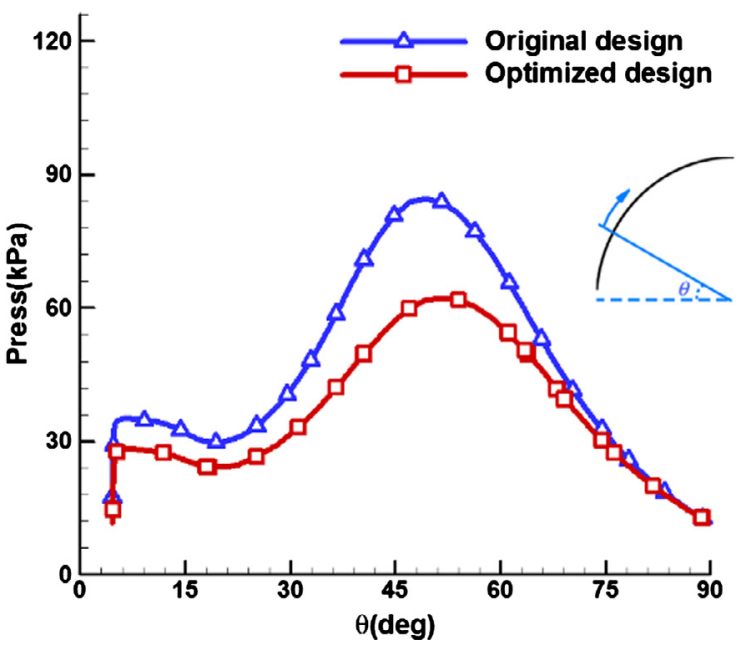

(a)Centerline surface pressure

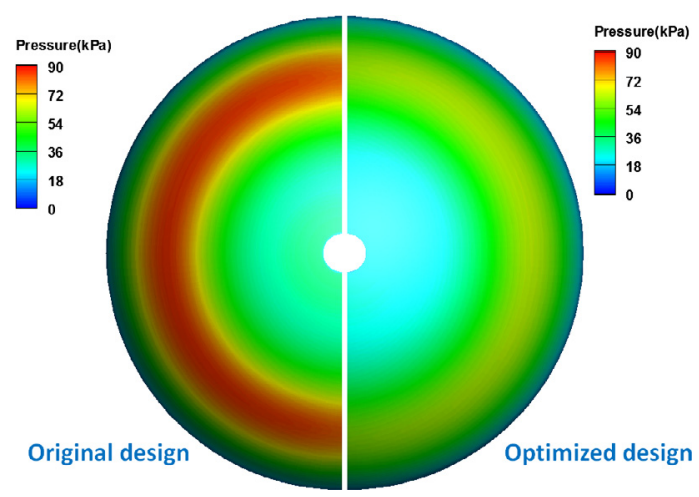

(b)Total surface pressure

Fig. 13. Comparisons of surface pressure distribution between original and optimized design.

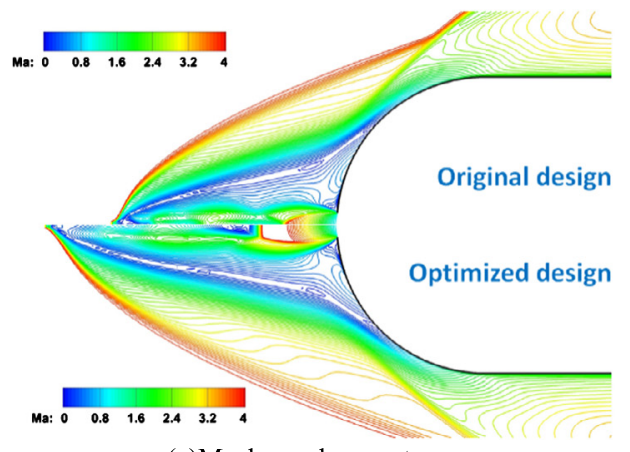

(a)Mach number contours

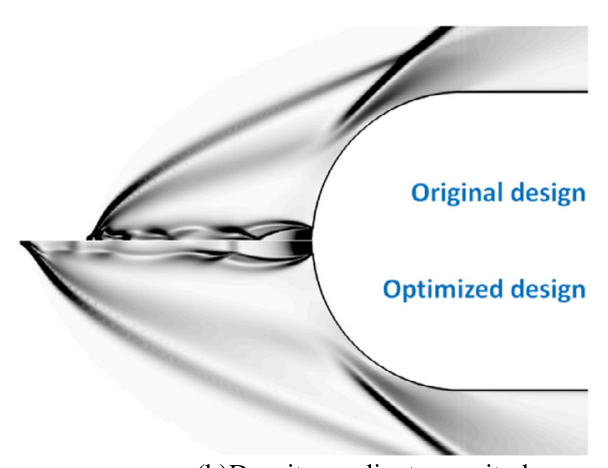

(b)Density gradient magnitudes

Fig. 14. Comparisons of flow field structure between original and optimized design.

(2) The optimized solutions for the multi-objective design optimization are obtained, which show reasonable agreement with the results obtained by the computational fluid dynamics. The optimization solutions show that both drag reduction factor $R_{\mathrm{d}}$ and drag reduction effectiveness $E_{\text {eff }}$ further increase. However, the tradeoff is needed between the two optimization objectives.

(3) The drag reduction factor $R_{\mathrm{d}}$ further increases by $28.16 \%$, whereas drag reduction effectiveness $E_{\text {eff }}$ also further increases by $116.47 \%$. At the same time, for the optimized design, the location for the region of energy deposition appears to be the maximized distance between the nose of the blunt body and the energy spot. Therefore, the jet has a stronger penetration in the optimized control configuration.

The thermal protection is the other important process during the design of the supersonic/hypersonic vehicles, but the drag and heat release reductions on vehicles are often conflicting, and they should be addressed in the future study.

\section{Declaration of competing interest}

We declare that we have no financial and personal relationships with other people or organizations that can inappropriately influence our work, there is no professional or other personal interest of any nature or kind in any product, service and/or company that could be construed as influencing the position presented in the manuscript entitled, "Multi-objective design optimization of the combinational configuration of the upstream energy deposition and opposing jet for drag reduction in supersonic flows".

\section{Acknowledgements}

The authors are very grateful to Professor Chao Yan from Beihang University for valuable discussions. This work is supported by the Postdoctoral Project for Advanced Engineering Construction of the Chinese Academy of Sciences. 


\section{References}

[1] W. Huang, A survey of drag and heat reduction in supersonic flows by a counterflowing jet and its combinations, J. Zhejiang Univ. Sci. A 16 (7) (2015) $551-561$.

[2] W. Huang, J. Liu, Z.X. Xia, Drag reduction mechanism induced by a combinational opposing jet and spike concept in supersonic flows, Acta Astronaut. 115 (2015) 24-31.

[3] J. Guo, G. Lin, X. Bu, H. Li, Parametric study on the heat transfer of a blunt body with counterflowing jets in hypersonic flows, Int. J. Heat Mass Transf. 121 (2018) 84-96.

[4] Kang Zhong, Chao Yan, Shu-Sheng Chen, Tian-Xin Zhang, Shuai Lou, Aerodisk effects on drag reduction for hypersonic blunt body with an ellipsoid nose, Aerosp. Sci. Technol. 86 (2019) 599-612.

[5] V. Menezes, S. Saravanan, G. Jagadeesh, K.P. Reddy, Experimental investigations of hypersonic flow over highly blunted cones with aerospikes, AIAA J. 41 (10) (2003) 1955-1966.

[6] J. Gopalan, V. Menezes, K. Reddy, T. Hashimoto, M. Sun, K. Takayama, Hypersonic buzz phenomenon on the spiked blunt cones, in: AIAA 41st Aerospace Sciences Meetings, 6-9 January 2003, Reno, USA, Reston.

[7] R. Zhang, W. Huang, Y. Li, C. Zheng, R. Moradic, Drag and heat flux reduction induced by the pulsed counterflowing jet with different waveforms on a blunt body in supersonic flows, Acta Astronaut. 160 (2019) 635-645.

[8] O.A. Azarova, D.D. Knight, Interaction of microwave and laser discharge resulting "heat spots" with supersonic combined cylinder bodies, Aerosp. Sci. Technol. 43 (2015) 343-349.

[9] T.A. Lapushkina, A.V. Erofeev, O.A. Azarova, O.V. Kravchenko, Interaction of a plane shock wave with an area of ionization instability of discharge plasma in air, Aerosp. Sci. Technol. 85 (2019) 347-358.

[10] Ahmad Rezaei Sangtabi, Abas Ramiar, Ali Akbar Ranjbar, M. Abdollahzadeh, Ali Kianifar, Influence of repetitive laser pulse energy depositions on supersonic flow over a sphere, cone and oblate spheroid, Aerosp. Sci. Technol. 76 (2018) $72-81$.

[11] Zhixiong Li, Tran Dinh Manh, M. Barzegar Gerdroodbary, Nguyen Dang Nam, R. Moradi, Houman Babazadeh, The influence of the wedge shock generator on the vortex structure within the trapezoidal cavity at supersonic flow, Aerosp. Sci. Technol. 98 (2020) 105695.

[12] Yicheng Li, M. Barzegar Gerdroodbary, R. Moradi, Houman Babazadeh, The influence of the sinusoidal shock generator on the mixing rate of multi hydrogen jets at supersonic flow, Aerosp. Sci. Technol. 96 (2020) 105579.

[13] Keivan Fallah, M. Barzegar Gerdroodbary, Atena Ghaderi, Javad Alinejad, The influence of micro air jets on mixing augmentation of fuel in cavity flameholder at supersonic flow, Aerosp. Sci. Technol. 76 (2018) 187-193.

[14] A. Hassanvand, M. Barzegar Gerdroodbary, Keivan Fallah, Rasoul Moradi, Effect of dual micro fuel jets on mixing performance of hydrogen in cavity flameholder at supersonic flow, Int. J. Hydrog. Energy 43 (2018) 9829-9837.

[15] M. Barzegar Gerdroodbary, Mojtaba Mokhtari, Keivan Fallah, H. Pourmirzaagha, The influence of micro air jets on mixing augmentation of transverse hydrogen jet insupersonic flow, Int. J. Hydrog. Energy 41 (2016) 22497-22508.

[16] A. Anazadehsayed, M. Barzegar Gerdroodbary, Y. Aminic, R. Moradi, Mixing augmentation of transverse hydrogen jet by injection of micro air jets in supersonic crossflow, Acta Astronaut. 137 (2017) 403-414.

[17] M. Barzegar Gerdroodbary, Keivan Fallah, H. Pourmirzaagha, Characteristics of transverse hydrogen jet in presence of multi air jets within scramjet combustor, Acta Astronaut. 132 (2017) 25-32.

[18] Rasoul Moradi, A. Mahyari, M. Barzegar Gerdroodbary, A. Abdollahi, Younes Amini, Shape effect of cavity flameholder on mixing zone of hydrogen jet at supersonic flow, Int. J. Hydrog. Energy 43 (2018) 16364-16372.

[19] D.H. Crawford, Investigation of the flow over a spike-nose hemisphere-cylinder at Mach number of 6.8, NASA TN D-118, December 1959.

[20] P.J. Finley, The flow of a jet from a body opposing a supersonic free stream, J. Fluid Mech. 26 (2) (1966) 337-368.

[21] K. Hayashi, S. Aso, Y. Tani, Experimental study on thermal protection system by opposing jet in supersonic flow, J. Spacecr. Rockets 43 (2006) 233-236.

[22] K. Kremeyer, K. Sebastian, C. Shu, Computational study of shock mitigation and drag reduction by pulsed energy lines, AIAA J. 44 (8) (2006) 1720-1731.

[23] O.A. Azarova, D.D. Knight, An approach of drag force decrease for combined cylinder AD bodies under the action of microwave and laser energy deposition, Aerosp. Sci. Technol. 64 (2017) 154-160.
[24] A. Khamooshi, T. Taylor, D.W. Riggins, Drag and heat transfer reductions in high-speed flows, AIAA J. 45 (10) (2007) 2401-2413.

[25] C.D. Marley, D.W. Riggins, Numerical study of novel drag reduction techniques for hypersonic blunt bodies, AIAA J. 49 (9) (2011) 1871-1882.

[26] D. Das, S. Desai, V. Kulkarni, H. Gadgil, Performance assessment of energy deposition based drag reduction technique for Earth and Mars flight conditions, Acta Astronaut. 159 (2019) 418-428.

[27] K. Hayashi, S. Aso, Y. Tani, Numerical study of thermal protection system by opposing jet, in: 43rd AIAA Aerospace Sciences Meeting and Exhibit, 10-13 January 2005, Reno, Nevada.

[28] B. Launder, D.B. Spalding, The numerical computation of turbulent flows, Comput. Methods Appl. Mech. Eng. 3 (2) (1974) 269-289.

[29] L.K. Sherrie, R.T. Biedron, C.L. Rumsey, CFL3D user's manual (version 5.0) NASA/TM-1998-208444, 1998.

[30] G.M. Ashwin, J. Bibin, Concentrated energy addition for active drag reduction in hypersonic flow regime, Acta Astronaut. 142 (2018) 221-231.

[31] S. Ju, C. Yan, X. Wang, Y. Qin, Z. Ye, Optimization design of energy deposition on single expansion ramp nozzle, Acta Astronaut. 140 (2017) 351-361.

[32] S. Ju, C. Yan, X. Wang, Y. Qin, Z. Ye, Effect of energy addition parameters upon scramjet nozzle performances based on the variance analysis method, Aerosp. Sci. Technol. 70 (2017) 511-519.

[33] F.R. Menter, Two-equation eddy-viscosity turbulence models for engineering applications, AIAA J. 32 (8) (1994) 1598-1605.

[34] J. Blazek, Computational Fluid Dynamics: Principles and Applications, second ed., Elsevier Ltd., Oxford, 2005.

[35] T. Osuka, E. Erdem, N. Hasegawa, R. Majima, T. Tamba, S. Yokota, A. Sasoh, K. Kontis, Laser energy deposition effectiveness on shock-wave boundary-layer interactions over cylinder-flare combinations, Phys. Fluids 26 (9) (2014) 096103.

[36] Halil Kaya, Hakan Tiftikçi, Ümit Kutluay, Evren Sakarya, Generation of surrogate-based aerodynamic model of an UCAV configuration using an adaptive co-Kriging method, Aerosp. Sci. Technol. 95 (2019) 105511.

[37] Jianyang Yu, Zhao Wang, Fu Chen, Jianing Yu, Cong Wang, Kriging surrogate model applied in the mechanism study of tip leakage flow control in turbine cascade by multiple DBD plasma actuators, Aerosp. Sci. Technol. 85 (2019) $216-228$.

[38] K. Deb, A. Pratap, S. Agarwal, T. Meyarivan, A fast and elitist multi-objective genetic algorithm: NSGA-II, IEEE Trans. Evol. Comput. 6 (2002) 182-197.

[39] W. Huang, Z. Wang, D.B. Ingham, L. Ma, M. Pourkashanian, Design exploration for a single expansion ramp nozzle (SERN) using data mining, Acta Astronaut. 83 (2013) 10-17

[40] W. Huang, J. Liu, L. Yan, L. Jin, Multi-objective design optimization of the performance for the cavity flameholder in supersonic flows, Aerosp. Sci. Technol. 30 (2013) 246-254.

[41] Jie Huang, Weixing Yao, Multi-objective design optimization of hypersonic spiked blunt body with opposing jet, J. Spacecr. Rockets 56 (5) (2019) 1553-1563.

[42] M.Y. Ahmed, N. Qin, Surrogate-based multi-objective aerothermodynamic design optimization of hypersonic spiked bodies, AIAA J. 50 (4) (2012) 797-810.

[43] A.A. Tucker, G.T. Hutto, C.H. Dagli, Application of design of experiments to flight test: a case study, J. Aircr. 47 (2) (2010) 458-463.

[44] G. Wang, Adaptive response surface method using inherited Latin hypercube design points, J. Mech. Des. 125 (2) (2003) 210.

[45] S. Ju, C. Yan, X. Wang, Y. Qin, Z. Ye, Sensitivity analysis of geometric parameters upon the aerothermodynamic performances of Mars entry vehicle, Int. J. Heat Mass Transf. 120 (2018) 597-607.

[46] S.H. Lee, M.E. Goddard, N.R. Wray, et al., A better coefficient of determination for genetic profile analysis, Genet. Epidemiol. 36 (3) (2012) 214-224.

[47] J.A. Cornell, Factors that influence the value of the coefficient of determination in simple linear and nonlinear regression models, Phytopathology 77 (1) (1987) 63-70.

[48] A. Zollanvari, E.R. Dougherty, Moments and root-mean-square error of the Bayesian MMSE estimator of classification error in the Gaussian model, Pattern Recognit. 47 (2014) 2178-2192.

[49] Jie Huang, Wei-Xing Yao, Multi-objective design optimization of blunt body with spike and aerodisk in hypersonic flow, Aerosp. Sci. Technol. 93 (2019) 105122.

[50] Yang Shen, Wei Huang, Tian-Tian Zhang, Li Yan, Parametric modeling and aerodynamic optimization of EXPERT configuration at hypersonic speeds, Aerosp. Sci. Technol. 84 (2019) 641-649. 